\title{
Precise Image Registration with Structural Similarity Error Measurement Applied to Superresolution
}

\author{
Mahmood Amintoosi, Mahmood Fathy, and Nasser Mozayani \\ Computer Engineering Department, Iran University of Science and Technology, Narmak, 16846-13114 Tehran, Iran \\ Correspondence should be addressed to Mahmood Amintoosi, mamintoosi@yahoo.com
}

Received 11 November 2008; Revised 5 February 2009; Accepted 22 May 2009

Recommended by Lisimachos P. Kondi

\begin{abstract}
Precise image registration is a fundamental task in many computer vision algorithms including superresolution methods. The well known Lucas-Kanade (LK) algorithm is a very popular and efficient method among the various registration techniques. In this paper a modified version of it, based on the Structural Similarity (SSIM) image quality assessment is proposed. The core of the proposed method is contributing the SSIM in the sum of squared difference, which minimized by LK algorithm. Mathematical derivation of the proposed method is based on the unified framework of Baker et al. (2004). Experimental results over 1000 runs on synthesized data validate the better performance of the proposed modification of LK-algorithm, with respect to the original algorithm in terms of the rate and speed of convergence, where the signal-to-noise ratio is low. In addition the result of using the proposed approach in a superresolution application is given.
\end{abstract}

Copyright (C) 2009 Mahmood Amintoosi et al. This is an open access article distributed under the Creative Commons Attribution License, which permits unrestricted use, distribution, and reproduction in any medium, provided the original work is properly cited.

\section{Introduction}

One of the most critical aspects of many applications in image processing and computer vision, including SuperResolution, is the accurate estimation of motion, also known as image registration. The Super-Resolution (SR) techniques fuse a sequence of low-resolution images to produce a higher resolution image. The low-resolution (LR) images may be noisy and blurred and have some displacements with each other. These methods utilize information from multiple observed images to achieve restoration at resolutions higher than that of the original data. It is widely recognized that the accuracy of motion estimation is arguably the limiting factor in Super-Resolution restoration performance [1, 2], and so any fruitful consideration of this problem promises significant returns.

In SR literatures a variety of registration approaches have been presented. They can be classified into two main approaches: feature-based methods and area-based methods. Usually the motion parameters can be roughly estimated by a feature-based method before being refined by an areabased method [3]. One of the famous registration method is the pioneering work of Lucas and Kanade [4]. This is an area-based method which is based on using of a Taylor series approximation of the images. The motion parameters are the unknowns in the approximation, and they can be computed from the set of equations that can be derived from this approximation. Recently Baker et al. [5] introduced a unified framework for Lucas-Kanade algorithm, and we will use their formulation for explaining our method in the rest of this paper.

Recent advances in Super-Resolution techniques show trends toward methods which consider some prior knowledge or models as the additional input of the SR algorithm $[3,6,7]$. The model-based approaches import plausible highfrequency textures from an image database into the HighResolution (HR) image. Based on the mentioned hypothesis, in [8], we described a method for increasing the resolution, using an HR training image, in which the entire of HR training image is mapped and fused onto LR image. Its registration stage is a feature-based method using SIFT keypoints, which sometimes leads to inaccurate mapping. In [9] we used the LK algorithm for refining the result of the mentioned feature-based registration stage and proposed a method for specifying magnification factor automatically. In this paper we proposed a new version of LK-algorithm 


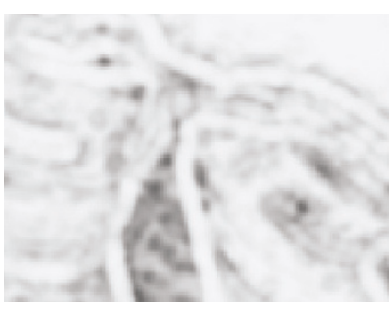

(h)

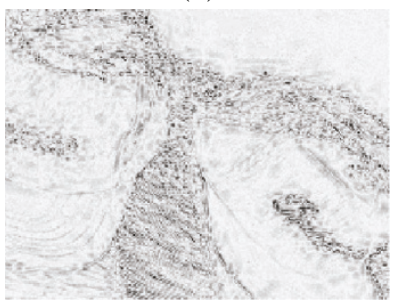

(k)

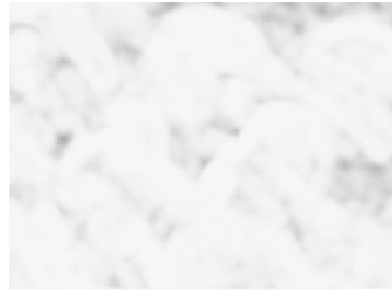

(i)

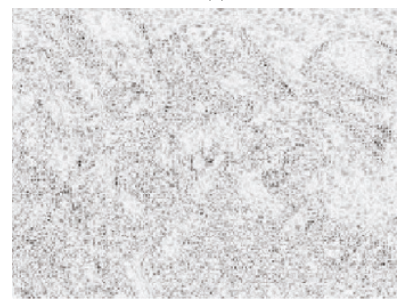

(1)
Figure 1: A portion of [10, Figure 7]. (h) and (i) are the contrast inverted of SSIM maps, and (k) and (l) are absolute error maps. The SSIM map shows that the structural differences are better than the other one. For the complete figure, please see Wang et al. [10].

which is better than its original form, when the LR image is under heavy noise. In the proposed method we used the Structural Similarity [10] as a weighting term to the objective function of LK algorithm. The chief idea of our approach is that the contrast-inverted form of SSIM shows the structural differences of two images, very better than absolute error map when the signal-to-noise is low. Experimental results show the better performance of the new variation of LKalgorithm with respect to its original form.

The rest of this paper is organized as follows. In Section 2 we first have a brief look at unifying framework of LK algorithm and Structural Similarity, which are the basis of the proposed method and then explain how to drive the Lucas-Kanade formulation based on SSIM. Section 3 provides the empirical validation of the proposed approach via experimental results with synthesized and real data. The last section is dedicated to the concluding Remarks.

\section{The Proposed Method}

We will use the unified framework of Baker et al. [5] for derivation of our extension to original LK-algorithm. Hence it is necessary to be familiar with the main parts of the unified framework, which is the subject of Section 2.1. Structural Similarity (SSIM) is introduced by Wang et al. [10] as a measurement for quality assessment of images. Section 2.2 is devoted to its summery and our definitions of Structural Dissimilarity (SDIS) based on it. The last subsection explains the proposed method in details.

Similar of SSIM map image, we define SDIS map image as the structural dissimilarity map of two images. More structural difference leads to higher value of SDIS.

2.1. LK-Algorithm, the Unified Framework. The goal of Lucas-Kanade is to align a template image $T(\mathbf{x})$ to an input image $I(\mathbf{x})$, by minimizing the following Sum of Squared Differences (SSDs) between two images:

$$
\operatorname{SSD}=\sum_{x}[I(\mathbf{W}(\mathbf{x} ; \mathbf{p}))-T(\mathbf{x})]^{2},
$$

where $\mathbf{W}(\mathbf{x} ; \mathbf{p})$ denote the parameterized set of allowed warps, $\mathbf{p}=\left(p_{1}, \ldots, p_{n}\right)^{T}$ is a vector of parameters, $I(\mathbf{W}(\mathbf{x} ; \mathbf{p}))$ is image $I$ warped back onto the coordinate frame of the template $T$, and $\mathbf{x}=(x, y)^{T}$ is a column vector containing the pixel coordinates. The warp $\mathbf{W}(\mathbf{x} ; \mathbf{p})$ takes the pixel $\mathbf{x}$ in the coordinate frame of the template $T$ and maps it to the subpixel location $\mathbf{W}(\mathbf{x} ; \mathbf{p})$ in the coordinate frame of the image $I[5]$. The warp model may be any transformation model such as affine, homography, or optical flow. But in this paper we concentrated on homography model. The minimization of the expression in (1) is performed with respect to $\mathbf{p}$, and the sum is performed over all of the pixels $\mathbf{x}$ in the template image $T$.

The Lucas-Kanade algorithm assumes that a current estimate of $\mathbf{p}$ is known and then iteratively solves for increments to the parameters $\Delta \mathbf{p}$; that is, the following expression is minimized with respect to $\Delta \mathbf{p}$, and then the parameters are updated:

$$
\begin{gathered}
\sum_{x}[I(\mathbf{W}(\mathbf{x} ; \mathbf{p}+\Delta \mathbf{p}))-T(\mathbf{x})]^{2}, \\
\mathbf{p} \leftarrow \mathbf{p}+\Delta \mathbf{p} .
\end{gathered}
$$

These two steps are iterated until the estimates of the parameters converge. $\Delta \mathbf{p}$ is calculated as follows:

$$
\Delta \mathbf{p}=H^{-1} \sum_{x}\left[\nabla I \frac{\partial \mathbf{W}}{\partial \mathbf{p}}\right]^{T}[T(\mathbf{x})-I(\mathbf{W}(\mathbf{x} ; \mathbf{p}))],
$$

where $H$ is the approximate Hessian matrix:

$$
H=\sum_{x}\left[\nabla I \frac{\partial \mathbf{W}}{\partial \mathbf{p}}\right]^{T}\left[\nabla I \frac{\partial \mathbf{W}}{\partial \mathbf{p}}\right],
$$

and $\nabla I=(\partial I / \partial x, \partial I / \partial y)$ is the gradient of image $I$ evaluated at $\mathbf{W}(\mathbf{x} ; \mathbf{p}), \partial \mathbf{W} / \partial \mathbf{p}$ is the Jacobian of the warp, and $\nabla I(\partial \mathbf{W} / \partial \mathbf{p})$ is the steepest descent images. For further details about the mentioned terms please see [5].

2.2. Error Measurement Based on SSIM. Mean Structural Similarity (MSSIM) for quality measurement introduced by Wang et al. [10] is defined as follows:

$$
\operatorname{MSSIM}(X, Y)=\frac{1}{M} \sum_{j=1}^{M} \operatorname{SSIM}\left(x_{j}, y_{j}\right),
$$

where $X$ and $Y$ are the reference and the distorted images, respectively, $x_{j}$ and $y_{j}$ are the image contents at the $j$ th local window, $M$ is the number of local windows of the image, and $\operatorname{SSIM}(x, y)$ is defined as follows:

$$
\operatorname{SSIM}(x, y)=\frac{\left(2 \mu_{x} \mu_{y}+C_{1}\right)\left(2 \sigma_{x y}+C_{2}\right)}{\left(\mu_{x}^{2}+\mu_{y}^{2}+C_{1}\right)\left(\sigma_{x}^{2}+\sigma_{y}^{2}+C_{2}\right)},
$$




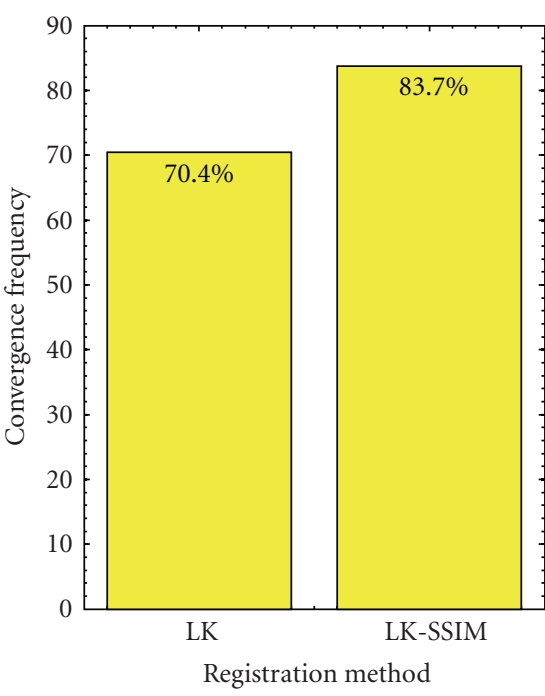

(a) Frequency of convergence

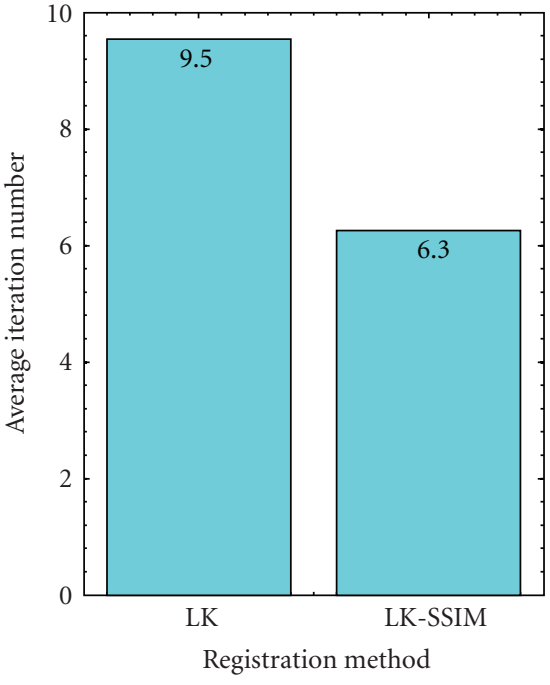

(b) Average number of cycles until convergence

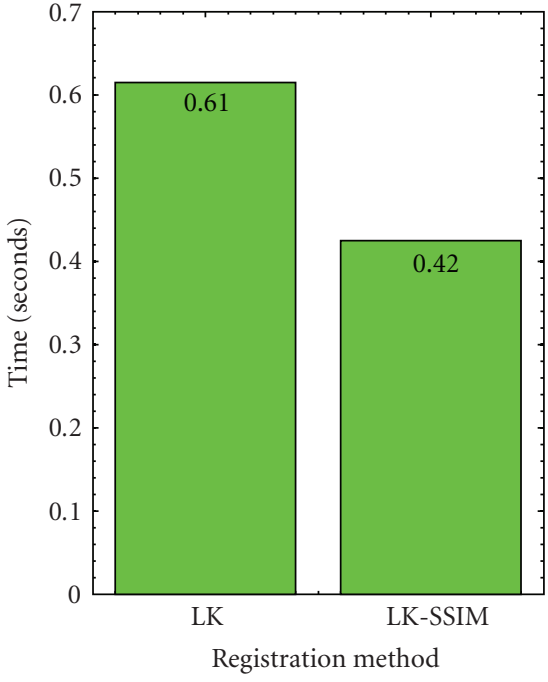

(c) Running time until convergence

FIGURE 2: The frequency of convergence, average number of cycles until convergence, and mean time of convergence over 1000 runs, with LK algorithm and the proposed method on "Takeo" dataset.

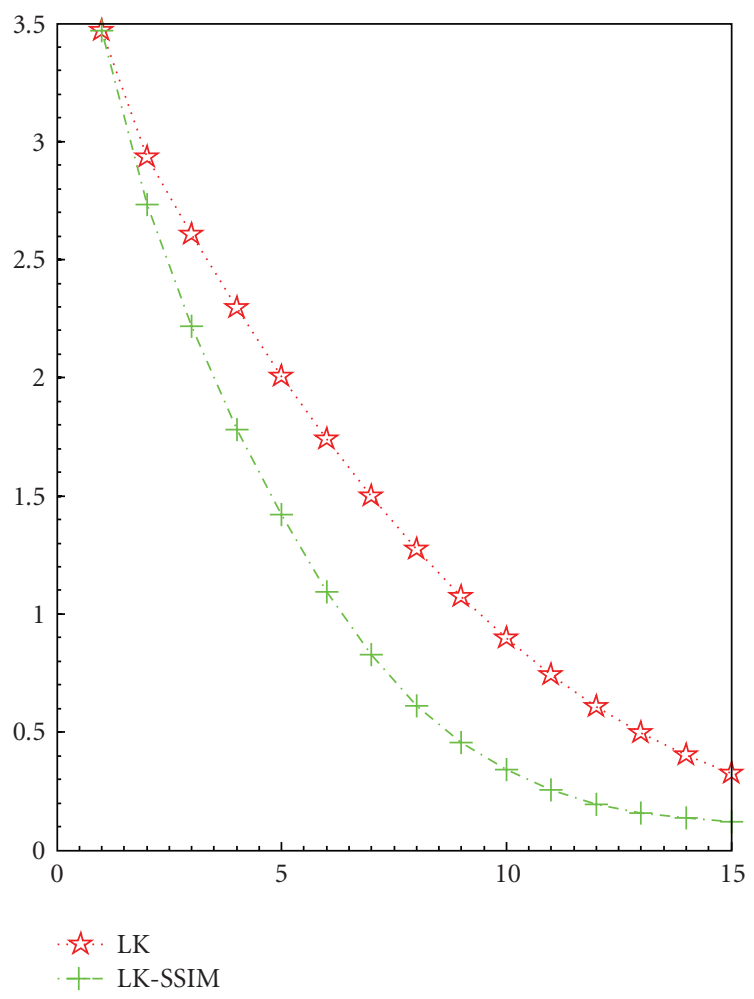

Figure 3: The average RMS Error over 1000 runs on "Takeo" dataset.

where $C_{1}$ and $C_{2}$ are some constants for avoiding instability; $\mu_{x}, \sigma_{x}$, and $\sigma_{x y}$ are estimates MSSIM of local statistics defined in Wang et al. [10]. The $\operatorname{MSSIM}(X, Y)$ is defined so that measurement similarity is closer to 1 when the images $X, Y$ are more similar. SSIM is defined for each pair of correspondence pixels. The image $Z$, which produced by

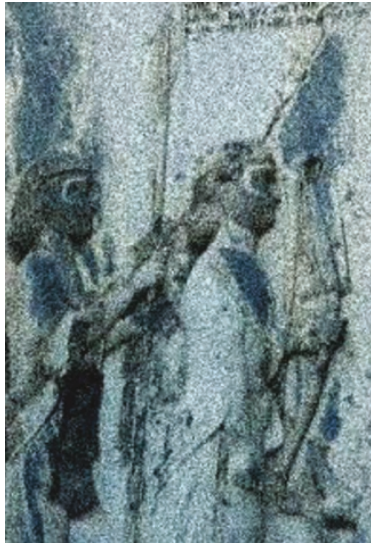

(a) The input LR image under heavy noise $(288 \times 196$ pixels $)$

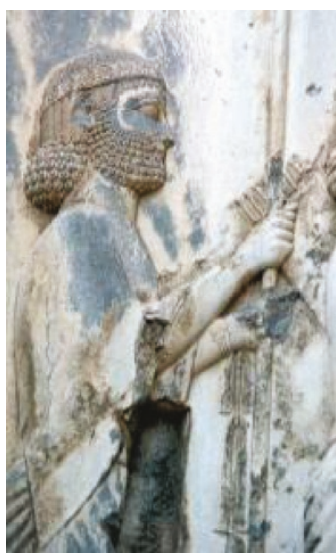

(b) HR image, with good quality, from the same scene but taken from different view points $(288 \times 176$ pixels $)$
Figure 4: Two images from bas relief of Darius. The goal is to enhance the region of the left image, corresponding to the right image. The resolution, view point, illumination, and color of two images are different.

computing the SSIM between each pixel pair, is named by Wang et al. [10] as SSIMmap image. An inversion or negative form of this criterion shows the structural differences of two images. This fact was mentioned by Wang et al. [10], where they compared the absolute error map and a contrast inverted SSIM map of two images. For clarity a portion of [10, Figure 7] is illustrated here in Figure 1. As can be seen, SSIM captures structural errors better than absolute error. Hence one can expect that contributing the SSIM onto the LK-algorithm's minimization function promises better result than its original form which is based on usual 


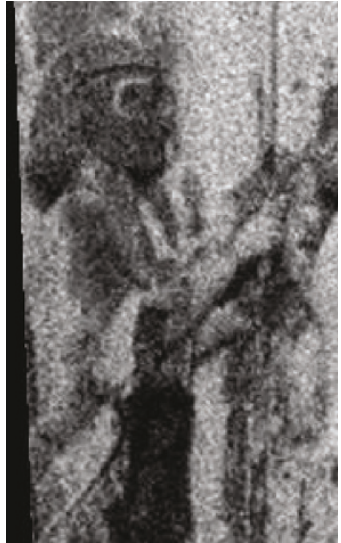

(a) $I(\mathbf{W}(\mathbf{x} ; \mathbf{p}))$

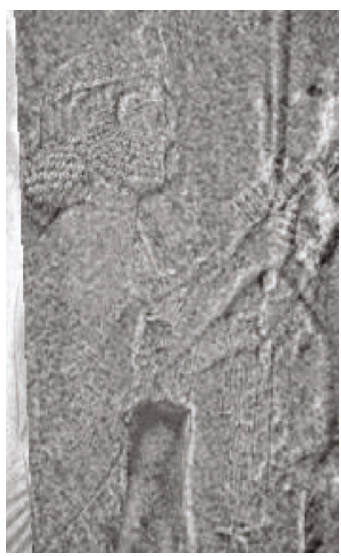

(c) Error image, $\mathbf{T}(\mathbf{x})-$ $I(\mathbf{W}(\mathbf{x} ; \mathbf{p}))$ in the first iteration

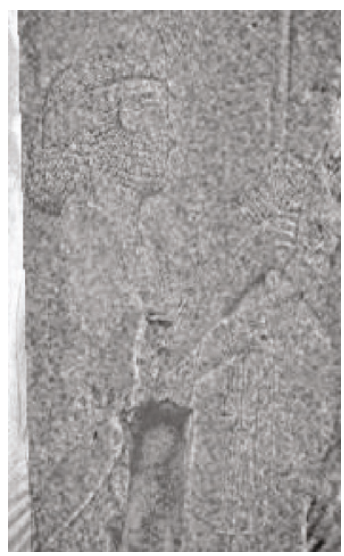

(e) Error image, $\mathbf{T}(\mathbf{x})$ $I(\mathbf{W}(\mathbf{x} ; \mathbf{p}))$ in the last iteration

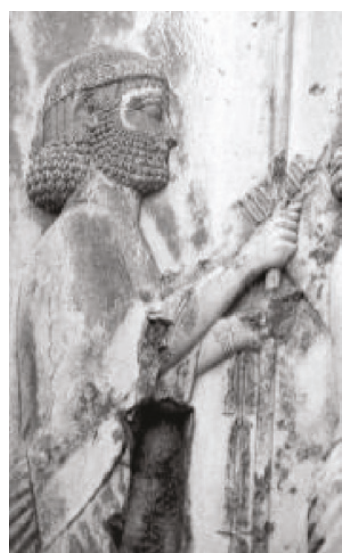

(b) Template (T)

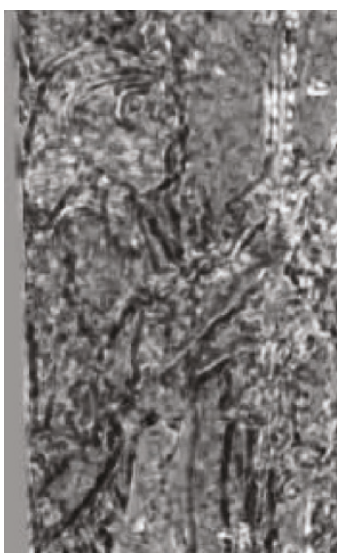

(d) SDIS error map image in the first iteration

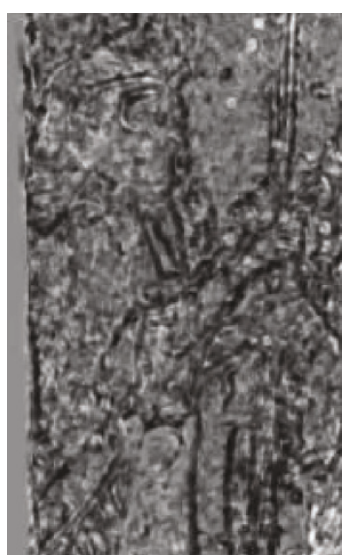

(f) SDIS error map image in the last iteration
FIGURE 5: Various intermediate results of executing the proposed method shown in Algorithm 1.

image difference. Among the various inverted forms of SSIM, such as "1/SSIM", "1-SSIM", and "-SSIM", we choose its negative form and called it SDIS as Structural Dissimilarity measurement:

$$
\operatorname{SDIS}(x, y)=-\operatorname{SSIM}(x, y) \text {. }
$$

2.3. Derivation of LK Algorithm Based on SDIS Map Image. In the proposed method, the defined error map, SDIS map imag,e is used as a weighting term of the error function. For convenience we call the SDIS map image of two images $I(\mathbf{W}(\mathbf{x} ; \mathbf{p}))$ and $T(\mathbf{x})$ as $E_{\text {SDIs. }}$ Hence our goal will be the minimization of the following function:

$$
\sum_{x} E_{\mathrm{SDIS}} \cdot[I(\mathbf{W}(\mathbf{x} ; \mathbf{p}))-T(\mathbf{x})]^{2},
$$

where dot denotes the element by element multiplication as “. *” operator in MATLAB. For minimizing (9) in an iterative manner similar to (2), we have to minimize the following function:

$$
\sum_{x} E_{\mathrm{SDIS}} \cdot[I(\mathbf{W}(\mathbf{x} ; \mathbf{p}+\Delta \mathbf{p}))-T(\mathbf{x})]^{2}
$$

where $E_{\text {SDIS }}$ is evaluated at $\mathbf{W}(\mathbf{x} ; \mathbf{p})$. Performing a first-order Taylor expansion on $I(\mathbf{W}(\mathbf{x} ; \mathbf{p}+\Delta \mathbf{p}))$ gives

$$
\mathrm{SSD}=\sum_{x} E_{\mathrm{SDIS}} \cdot\left[I(\mathbf{W}(\mathbf{x} ; \mathbf{p}))+\nabla I \frac{\partial \mathbf{W}}{\partial \mathbf{p}} \Delta \mathbf{p}-T(\mathbf{x})\right]^{2} .
$$

Finding the optimum value of $\Delta \mathbf{p}$ can be done by differentiating (11) with respect to $\Delta \mathbf{p}$, setting the result to equal zero and solving it:

$$
\begin{aligned}
\frac{\partial S S D}{\partial \Delta \mathbf{p}}= & 2 \sum_{x} E_{\text {SDIS }} \cdot\left[\nabla I \frac{\partial \mathbf{W}}{\partial \mathbf{p}}\right]^{T} \\
& \times\left[I(\mathbf{W}(\mathbf{x} ; \mathbf{p}))+\nabla I \frac{\partial \mathbf{W}}{\partial \mathbf{p}} \Delta \mathbf{p}-T(\mathbf{x})\right], \\
\frac{\partial S S D}{\partial \Delta \mathbf{p}}= & 0 \\
\Longrightarrow & \sum_{x} E_{\text {SDIS }} \cdot\left[\nabla I \frac{\partial \mathbf{W}}{\partial \mathbf{p}}\right]^{T} \nabla I \frac{\partial \mathbf{W}}{\partial \mathbf{p}} \Delta \mathbf{p} \\
& +\sum_{x} E_{\text {SDIS }} \cdot\left[\nabla I \frac{\partial \mathbf{W}}{\partial \mathbf{p}}\right]^{T}[I(\mathbf{W}(\mathbf{x} ; \mathbf{p}))-T(\mathbf{x})]=0
\end{aligned}
$$

Hence we have

$$
\Delta \mathbf{p}=H^{-1} \sum_{x} E_{\text {SDIS }} \cdot\left[\nabla I \frac{\partial \mathbf{W}}{\partial \mathbf{p}}\right]^{T}[T(\mathbf{x})-I(\mathbf{W}(\mathbf{x} ; \mathbf{p}))],
$$

where $H$ is

$$
H=\sum_{x} E_{\text {SDIS }} \cdot\left[\nabla I \frac{\partial \mathbf{W}}{\partial \mathbf{p}}\right]^{T}\left[\nabla I \frac{\partial \mathbf{W}}{\partial \mathbf{p}}\right] .
$$

The unified framework of Lucas-Kanade algorithm [5] is illustrated in Algorithm 1. In the original form of LK 
algorithm, $\Delta \mathbf{p}$ and the Hessian matrix were computed by (4) and (5), but in the proposed method, they are computed based on (13) and (14), respectively. For consistency with the unified framework, we have not described the computation of $E_{\text {SDIS }}$ needed in (13) and (14), explicitly in Algorithm 1.

Experimental results showed that the proposed method produced better results with respect to original LK algorithm, when the rate of signal-to-noise is low.

\section{Experimental Results}

In the first part of this section we will mention the experimental results for image registration using synthesized data. In the second part we will use the proposed method on an image superresolution application using real data.

3.1. Empirical Validation Using Synthesized Data. The experimental here has been done in a way similar to Baker et al. [5] Every synthesized experiment was done as in the following manner. A $100 \times 100$ pixel template $T(\mathbf{x})$ is manually selected from image $I(\mathbf{x})$. For producing a random projective warp $\mathbf{W}(\mathbf{x} ; \mathbf{p}), 4$ canonical points at the corners of the template are chosen, and then those points are randomly perturbed with additive white Gaussian noise of a certain variance. The warping model is computed with the method described in [11, Chapter 4]. Then $I(\mathbf{x})$ is warped with this model, and the two algorithms will run, starting from the identity warp.

Since 8 parameters in the projective warp have different units, the following error measure has been used rather than the errors in parameters. For each estimated warp, the RMS is computed over 4 canonical points of the distance between their current and correct locations.

We computed average RMS error, average frequency of convergence, average cycles needed, and average time taken until convergence over 1000 runs of randomly generated data. Before explaining the mentioned criteria used here, we describe our meaning of convergence. We say that an algorithm is converged if

(1) its last RMS error is smaller than its first error,

(2) after the last iteration the RMS error in canonical point locations is less than 1.0 pixels.

If an algorithm does not satisfy the second condition in its last iteration, it is considered as diverged even if allowing more iterations leads to RMS less than 1. In the following results, "Takeo" database of Baker et al. [5] has been used. The initial perturbation variance of canonical points was set to 4 pixels. Hence the initial RMS is always greater than 1 pixel, and thus the first condition is satisfied if the second condition is hold.

3.1.1. Frequency of Convergence. It is the percentage of runs, in which each algorithm converged over all 1000 runs. As can be seen in Figure 2(a), the proposed method converged more times than the original LK-algorithm. Note that LK stands for LK-algorithm, and LK-SSIM denotes the proposed method.
3.1.2. Average Number of Cycles Until Convergence. It is the average iterations needed until the convergence of each algorithm. The first iteration number in which the RMS of algorithm is below 1 pixel is considered as its number of cycles needed for convergence at that run. Figure 2(b) shows that in average the proposed method converges in fewer iterations. To avoid the results being biased by cases when one algorithm diverged, we included in the computation of this and the following criteria only those runs, which both of the two algorithms converged.

3.1.3. Running Time Until Convergence. The overhead for computing $E_{\mathrm{SDIS}}$ makes the running time of the proposed method longer than the LK-algorithm in each iteration. Thus for a predefined maximum iteration number, the LKalgorithm ends faster, but since the average number of cycles until convergence of our method is very less than the LKalgorithm, the average running time of our approach until convergence is smaller than that of LK-algorithm. Figure 2(c) shows the average running time of the two algorithms.

3.1.4. Average RMS Errors. The average RMS error is plotted over iteration numbers, for each method in Figure 3. Since all runs are performed on two specified images, averaging of RMS errors over all runs for each algorithm is meaningful. This value for each algorithm is its average RMS errors. As can be seen the proposed method is better.

The above results show the superior performance of the proposed method with respect to original LK-algorithm.

Our experimental results with other SNR values of image I showed that our approach is better than LK-algorithm when SNR is less than $30 \mathrm{~dB}$. Also we used some other images, and the results do not significantly differ with those reported here.

3.2. Superresolution Application. The proposed method can be used in every computer vision algorithm which requires image registration, such as panorama and super-resolution. Here, we tested the proposed method on a super-resolution problem in which the goal is to increase the resolution of some part of an LR image using an HR image. In many situations [9] someone may have an LR image or a video frame with low quality and a few HR images from some parts of the LR image with high quality. In this case he/she may desire to increase the quality or the resolution of his/her LR image using HR images. Consider the example shown in Figure 4; our goal is to enhance a region in noisy LR image 4(a), corresponding to HR image 4(b). The LR image is very noisy and color and resolution of images are different. The view point of two images has also slightly different. The LR and HR images in Figure 4(b) are our images $I$ and $T$ in Algorithm 1, respectively.

For enhancing the proper region of LR image, first we have to find an accurate transformation model for mapping HR image $T$ onto LR image $I$ and then fuse the resulting mapped image with LR image. This process is described in more details in [9]. With a feature-based stage a rough estimation of warp model is found, and the area-based 
Input: The reference image $I$ and template image $T$

Output: $\quad$ Registration parameters $\mathbf{p}=\left(p_{1}, \ldots, p_{n}\right)^{T}$ as the warp model $\mathbf{W}(\mathbf{x} ; \mathbf{p})$

(1) repeat

(2) Warp $I$ with $\mathbf{W}(\mathbf{x} ; \mathbf{p})$ to compute $I(\mathbf{W}(\mathbf{x} ; \mathbf{p}))$

(3) Compute the error image $T(x)-I(\mathbf{W}(\mathbf{x} ; \mathbf{p}))$

(4) Warp the gradient $\nabla I$ with $\mathbf{W}(\mathbf{x} ; \mathbf{p})$

(5) Evaluate the Jacobian $\partial \mathbf{W} / \partial \mathbf{p}$ at $(\mathbf{x} ; \mathbf{p})$

(6) Compute the steepest descent images $\nabla I(\partial \mathbf{W} / \partial \mathbf{p})$

(7) Compute the Hessian matrix using (14)

(8) Compute $[\nabla I(\partial \mathbf{W} / \partial \mathbf{p})]^{T}$ and $[T(x)-I(\mathbf{W}(\mathbf{x} ; \mathbf{p}))]$

(9) Compute $\Delta \mathbf{p}$ using $(13)$

(10) Update the parameters $\mathbf{p} \leftarrow \mathbf{p}+\Delta \mathbf{p}$

(11) until ||$\Delta \mathbf{p} \| \leq \varepsilon$ or Reaching to Maximum Iteration allowed

Algorithm 1: The Lucas-Kanade Algorithm using Structural Dissimilarity as a weighting term of error function.

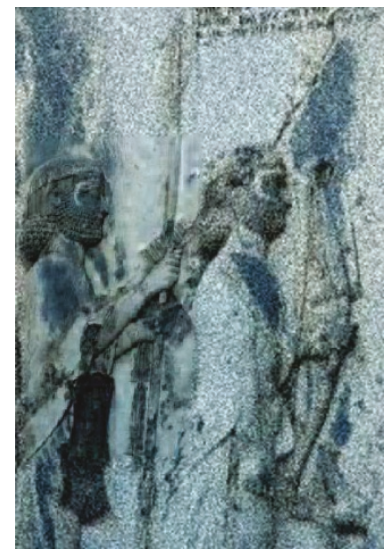

(a) LK

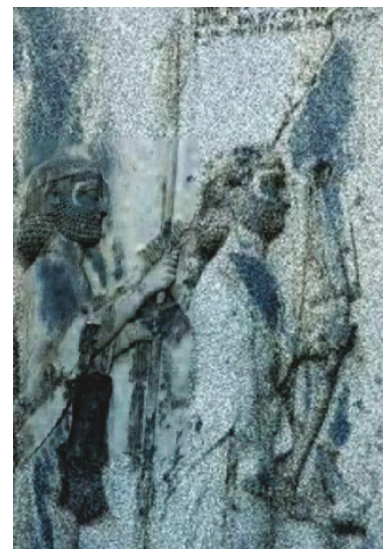

(b) LK-SSIM
FIGURE 6: Using LK-algorithm and LK-SSIM algorithm as areabased image registration stage of Amintoosi et al. [9] for enhancing the LR image 4(a) using HR image 4(b). A close-up demonstration is shown in Figure 7.

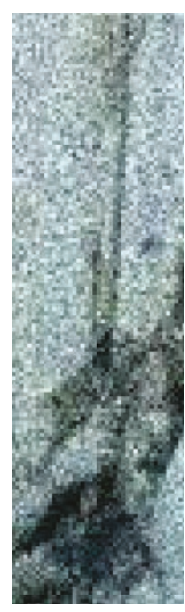

(a) Replication

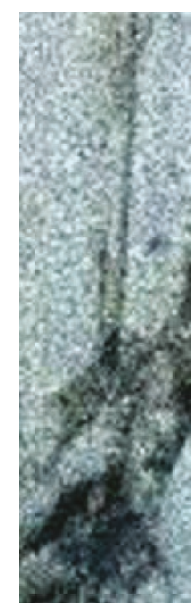

(b) Bicubic

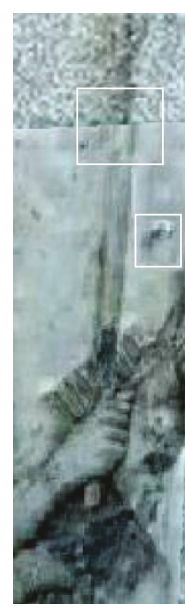

(c) LK

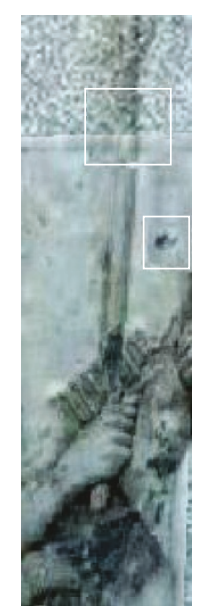

(d) LK-SSIM
FIGURE 7: Close-up of replication and bicubic resizing method, the method introduced in Amintoosi et al. [9] for enhancing the image shown in Figure 4(a) using HR image 4(b) with LK-algorithm and the proposed method as the area-based registration stage. stage tunes the result by a version of LK algorithm. The used feature-based stage is based on Lowe's [12] SIFT keypoints and Fischler and Bolles [13] RANSAC method. Here we compare the original LK-algorithm and the proposed modified version by using them as the tuning stage.

Figure 5 shows some intermediate results of Algorithm 1. Figure 5(a) shows the initial point of $I(\mathbf{W}(\mathbf{x} ; \mathbf{p}))$, in which $\mathbf{W}(\mathbf{x} ; \mathbf{p})$ is estimated by the feature-based registration stage for mapping 4(b) onto 4(a). Comparing Figures 5(c) and 5(d) clears that SDIS reduces the effect of noise, while preserving the structural differences of two images. In addition these images show that the most inaccuracy of initial warp model is about the upper-right area of the template, related to spear in the hand of the soldier. As can be seen SDIS error map Figure 5(d) highlighted these differences more better than usual difference (Figure 5(c)). Figures 5(e) and 5(f) show the mentioned error maps in the final iteration, in which the differences are reduced.

From our derivation of $\Delta \mathbf{p}$ and Hessian in (13) and (14), it is obvious that the proposed method benefits from original steepest descent images $\nabla I(\partial \mathbf{W} / \partial \mathbf{p})$ and SDIS information via $E_{\mathrm{SDIS}} \cdot \nabla I(\partial \mathrm{W} / \partial \mathbf{p})$.

Figure 6 shows the result of enhancing the LR image shown in Figure 4(a) using HR image 4(b) with the method proposed in Amintoosi et al. [9]. The magnification factor is set to 2. Figures 6(a) and 6(b) show the result when the LK-algorithm and the proposed method are used for the area-based registration stage. Here the blending stage is a combination of Wavelet fusion method [14] and multiband blending approach [15]. In these experiments the maximum iteration allowed is set to 15 . For enforcing the equal timing for two algorithms, the warping model returned by the proposed method in the appropriate iteration number (here 14 ) is used for reporting.

Figure 7 shows a subjective comparison between different methods on a magnified portion of their results. The proposed method (Figure $7(\mathrm{~d})$ ) produced the best result. In Figures $7(\mathrm{c})$ and $7(\mathrm{~d})$ the seamless blending approach has not been applied, to make the border of the fused regions more obvious. The better result of the proposed method is apparent by investigating the white boxes in two figures. 
It should be mentioned that the size of SSIM map image returned by Wang's implementation (available online at: http://www.cns.nyu.edu/ lcv/ssim/) is smaller than both of the two images. But for the proposed method (in (13) and (14)) it is necessary that the SDIS map image is equal to the size of each image. Hence we modified Wang's implementation according to our requirements.(available online at: http://webpages.iust.ac.ir/mamintoosi/Research.htm).

\section{Conclusion}

Feature-based and area-based methods are two broad categories in image alignment. When the ratio of signal-tonoise is very low, the feature-based approaches produce poor results, which can be refined by an area-based method. In this paper a new version of the famous area-based registration method, Lucas-Kanade algorithm, was proposed, which produces better results when the image is very noisy. The main idea of the proposed method is contributing SSIM, the Structural Similarity measurement of two images, into the formulation of LK-algorithm. Based on SSIM, a structural difference measurement, named as SDIS, was defined, which better reflects the dissimilarity of the two images compared to the usual image difference. The various objective comparisons showed that the proposed registration method outperforms the original LK-algorithm, in terms of convergence rate and speed. The subjective comparison in a superresolution problem in which the goal is to enhance an LR image with heavy noise using an HR image with good quality also showed the better performance of the proposed method.

\section{Acknowledgments}

The authors are indebted to two anonymous referees for valuable comments. They would also like to thank Dr. Peter Kovesi (School of Computer Science \& Software Engineering, The University of Western Australia, http:// www.csse.uwa.edu.au/) for his helpful MATLAB functions and Dr. Simon Baker, Dr. Ralph Gross and Dr. Iain Matthews for their registration package (http://www.cs.cmu .edu/ iainm/lk20.)

\section{References}

[1] S. Borman, Topics in multiframe superresolution restoration, Ph.D. thesis, University of Notre Dame, Notre Dame, Ind, USA, May 2004.

[2] R. R. Schultz and R. L. Stevenson, "Extraction of highresolution frames from video sequences," IEEE Transactions on Image Processing, vol. 5, no. 6, pp. 996-1011, 1996.

[3] T. Q. Pham, Spatiotonal adaptivity in super-resolution of undersampled image sequences, Ph.D. thesis, Technische Universiteit Delft, Delft, The Netherlands, 2006.

[4] B. D. Lucas and T. Kanade, "An iterative image registration technique with an application to stereo vision," in Proceedings of the International Joint Conference on Artificial Intelligence (IJCAI'81), vol. 2, pp. 674-679, 1981.
[5] S. Baker, R. Gross, and I. Matthews, "Lucas-Kanade 20 years on: a unifying framework," International Journal of Computer Vision, vol. 56, no. 3, pp. 221-255, 2004.

[6] S. Baker and T. Kanade, "Hallucinating faces," in Proceedings of the 4th IEEE International Conference on Automatic Face and Gesture Recognition (FG '00), p. 83, IEEE Computer Society, Washington, DC, USA, 2000.

[7] W. T. Freeman, T. R. Jones, and E. C. Pasztor, "Example-based super-resolution," IEEE Computer Graphics and Applications, vol. 22, no. 2, pp. 56-65, 2002.

[8] M. Amintoosi, M. Fathy, and N. Mozayani, "Reconstruction+synthesis: a hybrid method for multi-frame superresolution," in Proceedings of the 5th Iranian Conference on Machine Vision and Image Processing (MVIP '08), pp. 179-184, University of Tabriz, Tabriz, Iran, November 2008.

[9] M. Amintoosi, M. Fathy, and N. Mozayani, "Regional varying image super-resolution," in Proceedings of the IEEE International Joint Conference on Computational Sciences and Optimization (CSO '09), vol. 1, pp. 913-917, Sanya, China, April 2009.

[10] Z. Wang, A. C. Bovik, H. R. Sheikh, and E. P. Simoncelli, "Image quality assessment: from error visibility to structural similarity," IEEE Transactions on Image Processing, vol. 13, no. 4, pp. 600-612, 2004.

[11] R. I. Hartley and A. Zisserman, Multiple View Geometry in Computer Vision, Cambridge University Press, Cambridge, UK, 2nd edition, 2004.

[12] D. G. Lowe, "Object recognition from local scale-invariant features," in Proceedings of the IEEE International Conference on Computer Vision, vol. 2, pp. 1150-1157, IEEE Computer Society, Washington, DC, USA, 1999.

[13] M. A. Fischler and R. C. Bolles, "Random sample consensus: a paradigm for model fitting with applications to image analysis and automated cartography," Communications of the ACM, vol. 24, no. 6, pp. 381-395, 1981.

[14] P. Hill, N. Canagarajah, and D. Bull, "Image fusion using complex wavelets," in Proceedings of the British Machine Vision Conference (BMVC'02), pp. 487-496, 2002.

[15] P. J. Burt and E. H. Adelson, "A multiresolution spline with application to image mosaics," ACM Transactions on Graphics, vol. 2, no. 4, pp. 217-236, 1983. 